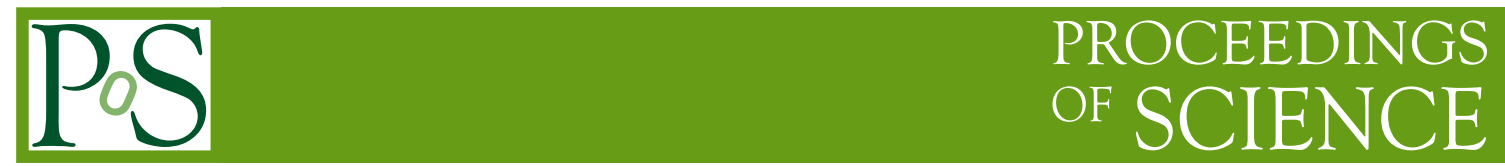

\title{
Lattice study of nuclear forces
}

\author{
Noriyoshi Ishii* \\ Department of Physics, The university of Tokyo \\ E-mail: ishii@ribf.riken.jp \\ for PACS-CS and HAL QCD Collaborations \\ Hadrons to Atomic nuclei
}

Recent progress of lattice QCD study of nuclear forces (potentials) is reviewed. Scattering phase shift is an important observable for two particle system. In lattice QCD, phase shifts are calculated from long distance behavior of Bethe-Salpeter (BS) wave functions by Lüscher's finite volume method. For applications to nuclear physics of multi-nucleon system, it is more advantageous to convert the information of phase shifts in the form of potentials. We therefore extend the method so as to generate the potentials from BS wave functions. These potentials are faithful to scattering phase shift by construction, because they can reproduce BS wave functions in which the information of phase shift is embeded in the long distance part.The method was first applied to the central potential in NN system. It is now applied to many objects, such as tensor potential, hyperon potentials, energy dependence of nuclear potentials, and investigations of the repulsive core at short distance.

The XXVII International Symposium on Lattice Field Theory - LAT2009

July 26-31 2009

Peking University, Beijing, China

${ }^{*}$ Speaker. 


\section{Introduction}

Scattering phase shift is an important physical observable in its own right. However, for nuclear physics, it is more advantageous to convert it into a form of the nuclear potential. Once such a nuclear potential is at our disposal, we can conveniently use it to study a variety of nuclear phenomena based on the effective degrees of freedom, i.e., the nucleons. It provides us with physics insights into the structures and the reactions of atomic nuclei, as well as the supernova explosion of type II and the structure of neutron stars through the equation of states of cold and dense nuclear matter. Enormous efforts in this line are integrated into a form of realistic nuclear potentials [1]. By using about $40-50$ adjustable parameters, they can reproduce several thousands of experimental $\mathrm{NN}$ data with $\chi^{2} / \mathrm{NDF} \sim 1$, which consist of the scattering phase shifts and the deuteron property. Also, the potentials from the chiral effective field theory attract an growing interest [2].

Unlike the nucleon sector, only a limited number of experimental information is available in the hyperon sector. This is due to the absence of accelerator facilities, which can generate direct hyperon beam. If there were a sufficient number of experimental information on the hyperonhyperon and hyperon-nucleon scatterings, they would be converted into realistic hyperon potentials, which could help us study the structure of hyper-nuclei and possible generation of hyperon matter in the neutron star core.

The standard method to obtain the scattering phase shifts in lattice QCD is Lüscher's finite volume method [3]. It can be used to provide QCD predictions/postdictions for the scattering phase shifts not only in the nucleon sector [4,5], but also in the hyperon sectors [6]. One may come up with a straightforward way to obtain realistic inter-baryon potentials in lattice QCD, i.e., sufficient number of scattering phase shifts are generated by Lüscher's method at the initial stage, which are converted to inter-baryon potentials with the help of the inverse scattering theory. However, this is difficult in practice, because it involves an infinite number of scattering phase shift at the initial stage. Thus, it is desirable to have a direct method to obtain realistic inter-baryon potentials in lattice QCD.

The method recently proposed by Ref. [7] is such a method. It can be given a background in terms of Lüscher's finite volume method. By using the effective Schrödinger equation, it constructs nuclear potentials from the Bethe-Salpeter (BS) wave functions generated by lattice QCD. Since the information of the scattering phase shift is embedded in the long distance part of the BS wave functions, it is possible to generate a realistic nuclear potential, which reproduces the QCD predictions of the scattering phase shift extracted by Lüscher's method.

In this paper, after a brief review of the general idea how to construct a realistic nuclear potential in lattice QCD, we present quenched QCD results of the central and the tensor potentials obtained at the leading order of the derivative expansion. After the discussion of the convergence of the derivative expansion, we give dynamical QCD results by using $2+1$ flavor gauge configurations generated by PACS-CS Collaboration. Finally, we mention the hyperon potentials ( $N \Xi$ and $N \Lambda$ ), to which our method can be equally applied.

\section{General idea to construct nuclear potential in QCD}

We consider (equial-time) Bethe-Salpeter (BS) wave function for two nucleons in the center 
of mass frame. By choosing particular composite interpolating fields $p(x)$ and $n(x)$ for proton and neutron, the BS wave function is defined as

$$
\psi_{\vec{k}}(\vec{x}-\vec{y}) \equiv\langle 0|p(\vec{x}) n(\vec{y})| p(\vec{k}) n(-\vec{k}), \text { in }\rangle=\lim _{t \rightarrow+0}\langle 0|T[p(\vec{x}, t) n(\vec{y}, 0)]| p(\vec{k}) n(-\vec{k}), \text { in }\rangle,
$$

where $\vec{k}$ denotes the asymptotic momentum of the proton relative to the neutron. It is related to the relativistic total energy of the state as $P_{0}=2 \sqrt{m_{N}^{2}+\vec{k}^{2}}$ with $m_{N}$ being the nucleon mass. Quite naively, this matrix element may be regarded as an amplitude to find three quarks at $\vec{x}$ and another three quarks at $\vec{y}$, where $p(\vec{x})$ and $n(\vec{y})$ are used to probe nucleons in the state $\mid p(\vec{k}) n(-\vec{k})$, in $\rangle$. At long distance $|\vec{x}-\vec{y}| \rightarrow$ large, it shows an asymptotic behavior, which is characterized by the scattering phase shift $\delta_{l}(k)$ in exactly the same way as a scattering wave function in the quantum mechanics as $[8,9,10]$

$$
\psi_{\vec{k}}(\vec{x}-\vec{y}) \simeq A \frac{\sin \left(k|\vec{x}-\vec{y}|-\pi l / 2+\delta_{l}(k)\right)}{k|\vec{x}-\vec{y}|}+\cdots .
$$

To prove this behavior, Nishijima-Zimmerman-Haag (NZH) reduction formula [11] is conveniently used. Note that any local composite nucleon field $N(x)$ leads to the same asymptotic behavior Eq. (2.2), as far as $N(x)$ has non-vanishing overlap with a single nucleon state, i.e., $\langle 0|N(x)| N\rangle \neq 0$. In Lüscher's finite volume method, which is the standard method to calculate the scattering phase shift in lattice QCD, the phase shift embedded in BS wave function in this manner is extracted from the energy spectrum in a finite periodic box [3]. (For explicit use of BS wave function in Lüscher's finite volume method, see Ref. [9].)

For nuclear physics, it is more advantageous to convert the data of the phase shift into a form of nuclear potentials. We therefore wish to extend Lüscher's method so as to obtain the nuclear potentials directly. For this purpose, we use the remarkable similarity in the asymptotic behaviors between the BS wave function Eq. (2.2) of QCD and the scattering wave function in the quantum mechanics. This similarity motivates us to construct a nuclear potential so that it can reproduce all the BS wave functions simultaneously in wide range of energy region. Then, the resulting potential can reproduce the phase shifts predicted by QCD. In this way, it becomes possible to construct realistic nuclear potentials by lattice QCD.

To proceed, we define the nuclear potential $U\left(\vec{r}, \vec{r}^{\prime}\right)$ by the effective Schrödinger equation [10]

$$
\left(\triangle+k^{2}\right) \psi_{\vec{k}}(\vec{r})=m_{N} \int d^{3} r^{\prime} U\left(\vec{r}, \vec{r}^{\prime}\right) \psi_{\vec{k}}\left(\vec{r}^{\prime}\right)
$$

where $m_{N}$ denotes the nucleon mass. We demand this equation to be simultaneously satisfied by $\psi_{\vec{k}}(\vec{r})$ in wide $\vec{k}$ region (or wide energy $2 \sqrt{m_{N}^{2}+\vec{k}^{2}}$ region). Note that $U\left(\vec{r}, \vec{r}^{\prime}\right)$ is most generally a non-local potential, and that, with our definition, $U\left(\vec{r}, \vec{r}^{\prime}\right)$ does not depend on the relativistic total energy $P_{0} \equiv 2 \sqrt{m_{N}^{2}+\vec{k}^{2}}$.

Several comments are in order.

(i) With our prescription, precise forms of potentials depend on particular choices of interpolating fields $p(x)$ and $n(x)$. However, even if their particular shapes are different, these potentials lead to the same phase shift. Remember that these potentials are constructed so as to give the phase shift obtained by Lüscher's method, which does not depend on a particular choice of interpolating fields. The situation is analogous to the unitary transformation in quantum mechanics, i.e., 
shapes of potentials are changed by unitary transformations without affecting any observables. The potentials, which lead to the same phase shift, are said to be phase-shift equivalent to each other.

(ii) Here, for simplicity, we do not pay attention to the orthogonality of the BS wave functions. In general, the BS wave functions are not expected to be orthogonal to each other with respect to the three-dimensional integral as $\int d^{3} x \psi_{\vec{k}^{\prime} ; \alpha \beta}^{*}(\vec{x}) \psi_{\vec{k} ; \alpha \beta}(\vec{x}) \neq \mathscr{N} \delta^{3}\left(\vec{k}-\vec{k}^{\prime}\right)$. If the violation of the orthogonality is serious, we have to take care of the orthogonalization to avoid non-hermitian potentials [10].

\section{The Derivative Expansion}

To construct the non-local potential $U\left(\vec{r}, \vec{r}^{\prime}\right)$ in Eq. (2.3), it is necessary to generate infinite number of BS wave functions, which is difficult in lattice QCD. The reason is two fold. (i) The energy spectrum is discretized in a finite spatial box. (ii) Although there is a method to access excited states in lattice QCD, it becomes the more difficult to access the higher excited states. Thus, we need an approximation, which enables us to construct $U\left(\vec{x}, \vec{x}^{\prime}\right)$ with a limited number of BS wave functions. For this purpose, we use the derivative expansion. We can start with the leading local potentials, and then take into account possible non-local terms (potentials, which contain derivatives) order by order. If the non-locality effect appears to be large, the convergence can be improved by changing the interpolating fields $p(x)$ and $n(y)$ in the sink side.

To proceed, we impose general requirements on the non-local potential $U\left(\vec{x}, \vec{x}^{\prime}\right)$ arising from the translational invariance, Galilean invariance, symmetry condition (identical particle condition), spatial rotation, spatial reflection, time-reversal invariance and hermiticity. The most general form has been derived in Ref. [12], to which we apply the derivative expansion. We are left with

$$
\begin{aligned}
U^{I}\left(\vec{x}, \vec{x}^{\prime}\right) & =V_{N N}^{I}(\vec{x}, \vec{\nabla}) \delta^{3}\left(\vec{x}-\vec{x}^{\prime}\right) \\
V_{N N}^{I}(\vec{x}, \vec{\nabla}) & =V_{0}^{I}(r)+V_{\sigma}^{I}(r) \vec{\sigma}_{1} \cdot \vec{\sigma}_{2}+V_{T}^{I}(r) S_{12}+V_{L S}^{I}(r) \vec{L} \cdot \vec{S}+O\left(\nabla^{2}\right),
\end{aligned}
$$

where $I$ indicates the total iso-spin of the two nucleon system. $\sigma_{1}$ and $\sigma_{2}$ act on the spin indices of the first and the second nucleons, respectively. $S_{12} \equiv 3\left(\vec{\sigma}_{1} \cdot \vec{x}\right)\left(\vec{\sigma}_{2} \cdot \vec{x}\right) / \vec{x}^{2}-\vec{\sigma}_{1} \cdot \vec{\sigma}_{2}$ is referred to as the tensor operator. $\vec{L} \equiv i \vec{x} \times \vec{\nabla}$ denotes the orbital angular momentum operator. $\vec{S} \equiv\left(\vec{\sigma}_{1}+\vec{\sigma}_{2}\right) / 2$ denotes the total spin operator. Since $\vec{\sigma}_{1} \cdot \vec{\sigma}_{2}$ reduces to 1 for spin triplet, and -3 for spin singlet, $V_{0}(r)+V_{\sigma}(r) \vec{\sigma}_{1} \cdot \vec{\sigma}_{2}$ is conveniently combined into the form of the "central potential" $V_{C}(r)$ as

$$
V_{C}\left(r ;{ }^{1} S_{0}\right) \equiv V_{0}(r)-3 V_{\sigma}(r), \quad V_{C}\left(r{ }^{3} S_{1}\right) \equiv V_{0}(r)+V_{\sigma}(r) .
$$

$V_{T}(r)$ and $V_{L S}(r)$ are referred to as the tensor potential and the LS potential, respectively. Note that $V_{C}(r), V_{T}(r)$ and $V_{L S}(r)$ play important roles in conventional nuclear physics.

At the leading order, we truncate the nuclear potential by neglecting all the derivative terms as

$$
V_{N N}(\vec{x}, \vec{\nabla})=V_{C}(r)+V_{T}(r) S_{12}+O(\vec{\nabla}) .
$$

We insert this into the effective Schrödinger equation Eq. (2.3) to have

$$
\left(-\frac{\triangle}{m_{N}}+V_{C}(r)+V_{T}(r) S_{12}\right) \psi_{\vec{k}}(\vec{r})=\frac{k^{2}}{m_{N}} \psi_{\vec{k}}(\vec{r}) .
$$


Note that the reduced mass of two nucleon system is $\mu \equiv m_{N} / 2$. For $J^{P}=0^{+}\left({ }^{1} S_{0}\right), I=1$ channel, a further reduction is possible. Since $S_{12}$ acts as zero on wave functions in ${ }^{1} S_{0}$ channel, we can rewrite it as

$$
V_{C}\left(r,{ }^{1} S_{0}\right)=\frac{k^{2}}{m_{N}}+\frac{1}{m_{N}} \frac{\triangle \psi_{\vec{k}}(\vec{r})}{\psi_{\vec{k}}(\vec{r})} .
$$

We use this formula to obtain the central potential in ${ }^{1} S_{0}$ channel at the leading order of the derivative expansion. For $J^{P}=1^{+}\left({ }^{3} S_{1}-{ }^{3} D_{1}\right.$ coupled system) $I=0$ channel (the deuteron channel), the procedure is slightly involved, which is to be considered in Sect. 6.

At the next to leading order, we include the terms, which contains a single derivative, i.e., $V_{L S}(r) \vec{L} \cdot \vec{S}$ as

$$
V_{N N}(\vec{x}, \vec{\nabla})=V_{C}(r)+V_{T}(r) S_{12}+V_{L S}(r) \vec{L} \cdot \vec{S}+O\left(\vec{\nabla}^{2}\right) .
$$

This is inserted into the effective Schrödinger equation Eq. (2.3). Note that the action of $\vec{L} \cdot \vec{S}$ on $J^{P}=0^{+}\left({ }^{1} S_{0}\right)$ channel vanishes. Therefore, the formula Eq. (3.5) does not change at this order. In contrast, $\vec{L} \cdot \vec{S}$ gives a non-vanishing contribution to $J^{P}=1^{+}\left({ }^{3} S_{1}-{ }^{3} D_{1}\right)$ channel. Hence, the formula to calculate $V_{C}(r)$ and $V_{T}(r)$ is modified. We need an additional BS wave function to obtain these three potentials at this order.

At the next to next to leading order, we include $O\left(\vec{\nabla}^{2}\right)$ terms in the potential, which is inserted to the effective Schrödinger equation Eq. (2.3). To obtain these potentials, we need further BS wave functions. We perform this procedure repeatedly to obtain higher derivative terms by using increasing number of BS wave functions.

It is important to examine the convergence of the derivative expansion. The non-local potential is faithful to the scattering data in wide range of energy region, while it may not be so after the derivative expansion is applied. If the convergence appears to be unsatisfactory, improvement has to be done by changing interpolating field of nucleon.

\section{Lattice QCD setup}

We use quenched QCD unless otherwise indicated. We employ the standard plaquette gauge action with $\beta=5.7$ to generate gauge configurations on the lattice of the size $32^{3} \times N_{t}\left(N_{t}=32\right.$ and 48). The scale unit is introduced by rho meson mass in the chiral limit, which leads to the lattice spacing $a^{-1}=1.44(2) \mathrm{GeV}$ ( $a \simeq 0.137 \mathrm{fm}$ ) [4]. The spatial extension amounts to $L=32 a \simeq 4.4 \mathrm{fm}$. $1000-4000$ gauge configurations are used in our calculations. Quark propagators are generated by employing the standard Wilson quark action with the hopping parameters $\kappa=0.1640,0.1665$ and 0.1678, which correspond to $m_{\pi} \simeq 731,529$ and $380 \mathrm{MeV}$ and $m_{N} \simeq 1558,1334$ and $1197 \mathrm{MeV}$, respectively. Unless otherwise indicated, Dirichlet and periodic boundary conditions are imposed on quark fields along the temporal and spatial directions, respectively.

To obtain the BS wave function, we generate the four point correlator of the nucleon field as

$$
G(\vec{x}-\vec{y}, t) \equiv\langle 0|T[p(\vec{x}, t) n(\vec{y}, t) \overline{\mathbf{p}}(t=0) \overline{\mathbf{n}}(t=0)]| 0\rangle,
$$

where $p(x)$ and $n(x)$ denote local composite fields for proton and neutron, for which we employ the standard ones as $p(x) \equiv \varepsilon_{a b c}\left(u_{a}^{T}(x) C \gamma_{5} d_{b}(x)\right) u(x), n(x) \equiv \varepsilon_{a b c}\left(u_{a}^{T}(x) C \gamma_{5} d_{b}(x)\right) d(x)$. These 
fields are represented by Heisenberg picture in imaginary time. $\overline{\mathbf{p}}(t)$ and $\overline{\mathbf{n}}(t)$ denote interpolating fields for proton and neutron (wall source), i.e., $\overline{\mathbf{p}}(t) \equiv \sum_{\vec{x}, \vec{y}, \vec{z}} \varepsilon_{a b c} \bar{u}_{c}(\vec{x}, t)\left(\bar{d}_{b}(\vec{y}, t) C \gamma_{5} \bar{u}_{a}(\vec{z}, t)^{T}\right)$, $\overline{\mathbf{n}}(t) \equiv \sum_{\vec{x}, \vec{y}, \vec{z}} \varepsilon_{a b c} \bar{d}_{c}(\vec{x}, t)\left(\bar{d}_{b}(\vec{y}, t) C \gamma_{5} \bar{u}_{a}(\vec{z}, t)^{T}\right)$. Note that the total spatial momentum of the system vanishes because of the wall source. Therefore Eq. (4.1) becomes a function of $\vec{x}-\vec{y}$ due to the translational invariance. Eq. (4.1) for the large Euclidean time $t>0$ is dominated by the contribution from the lowest-lying state as

$$
G(\vec{x}-\vec{y}, t)=\sum_{n}\left\langle 0|p(\vec{x}, t) n(\vec{y}, t)| E_{n}\right\rangle\left\langle E_{n}|\overline{\mathbf{p}}(0) \overline{\mathbf{n}}(0)| 0\right\rangle=A_{0} e^{-E_{0} t} \psi_{E_{0}}(\vec{x}-\vec{y})+\cdots,
$$

where $E_{0}$ denotes the energy of the lowest lying state $\left|E_{0}\right\rangle$, and $A_{0} \equiv\left\langle E_{0}|\overline{\mathbf{p}}(0) \overline{\mathbf{n}}(0)| 0\right\rangle \cdot \psi_{E_{0}}(\vec{x}-\vec{y}) \equiv$ $\left\langle 0|p(\vec{x}) n(\vec{y})| E_{0}\right\rangle$ denotes the BS wave function. Needless to say, $\psi_{E_{0}}(\vec{r})$ does not depend on a particular choice of the interpolating fields in the source side, as long as $\left\langle E_{0}|\overline{\mathbf{p}} \overline{\mathbf{n}}| 0\right\rangle \neq 0$. (We use the wall source for the efficiency reason.) Quantum numbers of the wave function such as $J^{P}$ are controlled by quantum numbers of the interpolating fields in the source side. Because we adopted the wall source, $J^{P}=0^{+}$and $1^{+}$are obtained by combining the spins of $\overline{\mathbf{p}}$ and $\overline{\mathbf{n}}$.

\section{Central potential at the leading order of the derivative expansion}
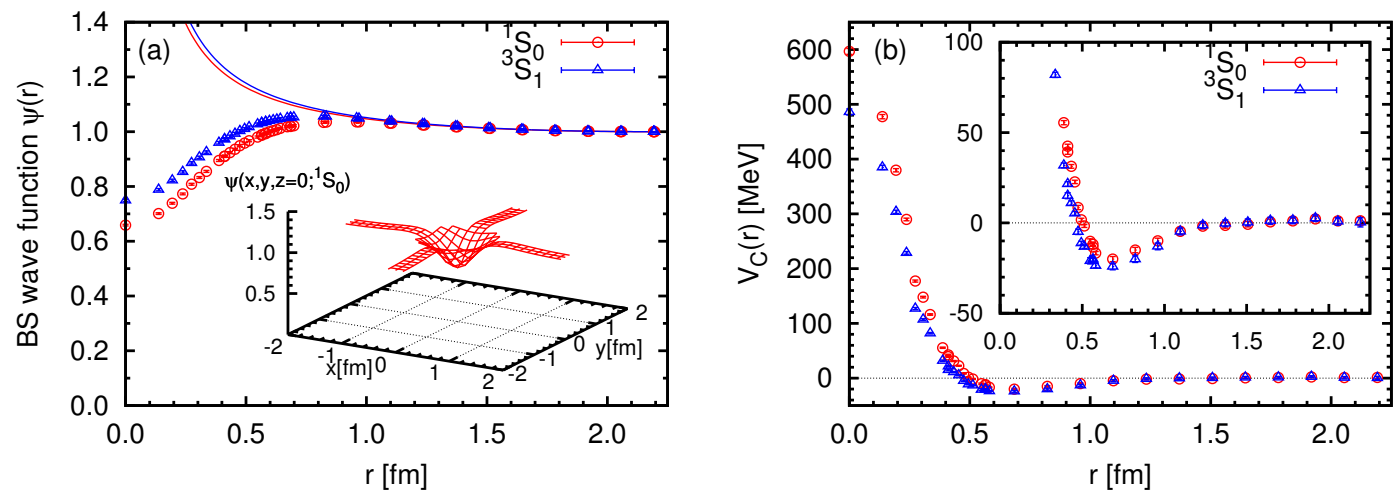

Figure 1: (a) BS wave functions in ${ }^{1} S_{0}$ and ${ }^{3} S_{1}$ channels for $m_{\pi} \simeq 529 \mathrm{MeV}$. The inset is a 3D plot of $\psi(x, y, z=0)$ for ${ }^{1} S_{0}$. The curves denote the results of the fits using the Green's function of Helmholtz equation in the region $11 a \leq r \leq 16 a$ and (b) The central potential in ${ }^{1} S_{0}$ channel and the effective central potential in ${ }^{3} S_{1}$ channel for $m_{\pi} \simeq 529 \mathrm{MeV}$.

Fig. 1(a) shows the quenched result of BS wave functions in ${ }^{1} S_{0}$ and ${ }^{3} S_{1}$ channels. To pick up "s-wave" component $\left({ }^{3} S_{1}\right)$ from the BS wave function in $J^{P}=1^{+}$channel, which is ${ }^{3} S_{1}-{ }^{3} D_{1}$ coupled system, we make a spatial average with respect to the cubic group $O$ as $\psi_{\alpha \beta}\left(\vec{r}^{3}{ }^{3} S_{1}\right) \equiv$ $\frac{1}{24} \sum_{g \in O} \psi_{\alpha \beta}(g \vec{r})$. Calculations are fully performed for $r \leq 0.7 \mathrm{fm}$, while, for $r \geq 0.7 \mathrm{fm}$, we restrict ourselves to the points on the coordinate axes and their nearest neighbors to reduce the calculational cost. (A rapid change of the potential is expected for $r \leq 0.7 \mathrm{fm}$, whereas a rather mild change is expected for $r \geq 0.7 \mathrm{fm}$.) We see that there are shrinks at short distance, which suggest the existence of repulsion. By using Eq. (3.5), the central potential $V_{C}(r)$ in ${ }^{1} S_{0}$ channel is constructed at the leading order. The result is shown in Fig. 1(b). 
To obtain the central potential in ${ }^{3} S_{1}$ channel, it is necessary to consider a coupled Schrödinger equations of ${ }^{3} S_{1}$ and ${ }^{3} D_{1}$ channels, which will be discussed in Sect. 6 . Here, we simply apply the same formula Eq. (3.5) to the wave function in ${ }^{3} S_{1}$ channel. Note that the resulting central potential can reproduce the ${ }^{3} S_{1}$ wave function without involving the tensor potential. Such a central potential is referred as the "effective central potential", in which the effect of the tensor potential is embedded implicitly. The result is also shown in Fig. 1(b).

In Fig. 1(b), we wee that phenomenological properties of the central nuclear potentials are reproduced. A repulsive core at short distance is surrounded by an attraction at medium distance. The effective central potential in ${ }^{3} S_{1}$ channel tends to be more attractive than the central potential in ${ }^{1} S_{0}$ channel. This is desirable for the existence of a bound state (deuteron) in ${ }^{3} S_{1}$ channel in reality. (No bound state exists in ${ }^{1} S_{0}$ channel.)

The non-relativistic energy $E \equiv \vec{k}^{2} / m_{N}$ in Eq. (3.5) is obtained by making a fit of BS wave functions with Green's function of Helmholtz equation defined as

$$
\left(\triangle+k^{2}\right) G\left(\vec{x} ; k^{2}\right)=-\delta_{L}(\vec{x}),
$$

where $\delta_{L}(\vec{x}) \equiv \sum_{\vec{n} \in \mathbb{Z}^{3}} \exp (2 \pi i \vec{n} \cdot \vec{x} / L)$ denotes the periodic delta function in the three dimensional torus of spatial extension $L$. The fit is performed in the region $11 a \lesssim r \lesssim 16 a$, where the interaction is seen to become negligible from a plot of $\triangle \psi(\vec{x}) / \psi(\vec{x})$ [9]. The resulting non-relativistic energies are quite small, i.e., $E=-0.509$ (94) $\mathrm{MeV}$ for ${ }^{1} S_{0}$ channel and $E=-0.560(110) \mathrm{MeV}$ for ${ }^{3} S_{1}$ channel. Note that negative $E$ does not necessarily mean a formation of a bound state. This is because two nucleons cannot be separated from each other beyond the range of interaction in a finite volume. The result is also shown in Fig. 1(a), where Green's functions along the coordinate axis are plotted with solid lines.
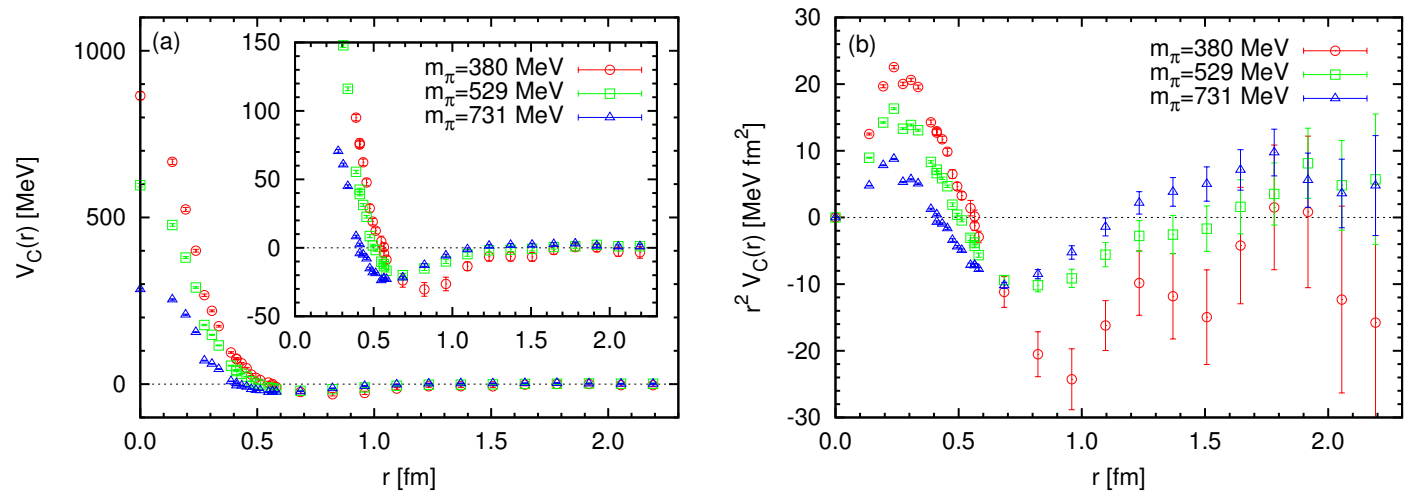

Figure 2: (a) The central potential in ${ }^{1} S_{0}$ channel for three quark masses and (b) those with $r^{2}$ multiplied.

Fig. 2(a) shows the central potentials in ${ }^{1} S_{0}$ channel at the leading order for three values of the quark mass. To see the strength of the potentials more accurately, we plot them with a factor $r^{2}$ in Fig. 2(b) to reflect the effect of three-dimensional volume element. We see that, as the quark mass decreases, the repulsive core at short distance grows rapidly, and the attraction at medium distance is enhanced gradually. The enhancement of the attraction is natural, since the smaller quark mass enables the virtual pion to propagate the longer distance. These results suggest the importance of direct lattice QCD calculations in the light quark mass region. 


\section{Tensor Potential at the leading order of the derivative expansion}

In order to construct the central and the tensor potentials, it is necessary to take into account the coupling of s-wave $(l=0)$ and d-wave $(l=2)$ components of BS wave function in $J^{P}=1^{+}(I=0)$ channel. Note that the coupling is induced by the tensor potential, due to which the deuteron is generated as a bound state in reality. The tensor potential plays a key role in the stability of atomic nuclei and the saturation of nuclear matter density. Its importance at short distance is pointed out recently by experimental studies of Short Ranged Correlated (SRC) nucleon pair, which may affects the structure of the cold dense nuclear system such as neutron stars [13]. However, the experimental determination of tensor potential at short distance is difficult, because it appears on top of the repulsive core and the centrifugal barrier.

Construction of tensor potential depends on the quality of d-wave wave function on the lattice. On the lattice, we construct a BS wave function in $T_{1}^{+}$representation of the cubic group, which corresponds to $J^{P}=1^{+}$up to $J \geq 4$ contamination. We decompose it into orbitally $A_{1}^{+}$part $\psi^{(S)}(\vec{r})$, which corresponds to s-wave up to $l \geq 4$ contamination, and orbitally non- $A_{1}^{+}$part $\psi^{(D)}(\vec{r})$. The decomposition is performed by using the projection operators $P$ and $Q$ defined as

$$
\psi_{\alpha \beta}^{(S)}(\vec{r})=P[\psi]_{\alpha \beta}(\vec{r}) \equiv \frac{1}{24} \sum_{g \in O} \psi_{\alpha \beta}(g \vec{r}), \quad \psi_{\alpha \beta}^{(D)}(\vec{r})=Q[\psi]_{\alpha \beta}(\vec{r}) \equiv \psi_{\alpha \beta}(\vec{r})-\psi_{\alpha \beta}^{(S)}(\vec{r})
$$

where $O$ denotes the cubic group with 24 elements. From the orthogonality relations of the characters of the representations, we find that the orbital part of $\psi^{(D)}(\vec{r})$ consists of either $E^{+}, T_{2}^{+}$or $T_{1}^{+}$ representations. $E^{+}$and $T_{2}^{+}$correspond to d-wave up to $l \geq 4$ contamination. Orbital $T_{1}^{+}$representation corresponds to g-wave $(l=4)$ up to $l \geq 6$, which enters through $J^{P}=4^{+}$component of $A_{1}^{+}$ through the relation $4^{+}\left(J^{P}\right)=1$ (spin) $\otimes 4^{+}$(orbital). Fig. 3(b) shows BS wave functions for $T_{1}^{+}$ $\left(J^{P} \simeq 1^{+}\right)$and the azimuthal quantum number $M=0$. We see that $\psi^{(S)}$ is single-valued, whereas $\psi^{(D)}$ 's are multi-valued. Since the angular dependence manifests itself as multi-valuedness, it follows that $\psi^{(S)}$ is dominated by s-wave contribution. To consider $\psi^{(D)}$, we note that d-wave
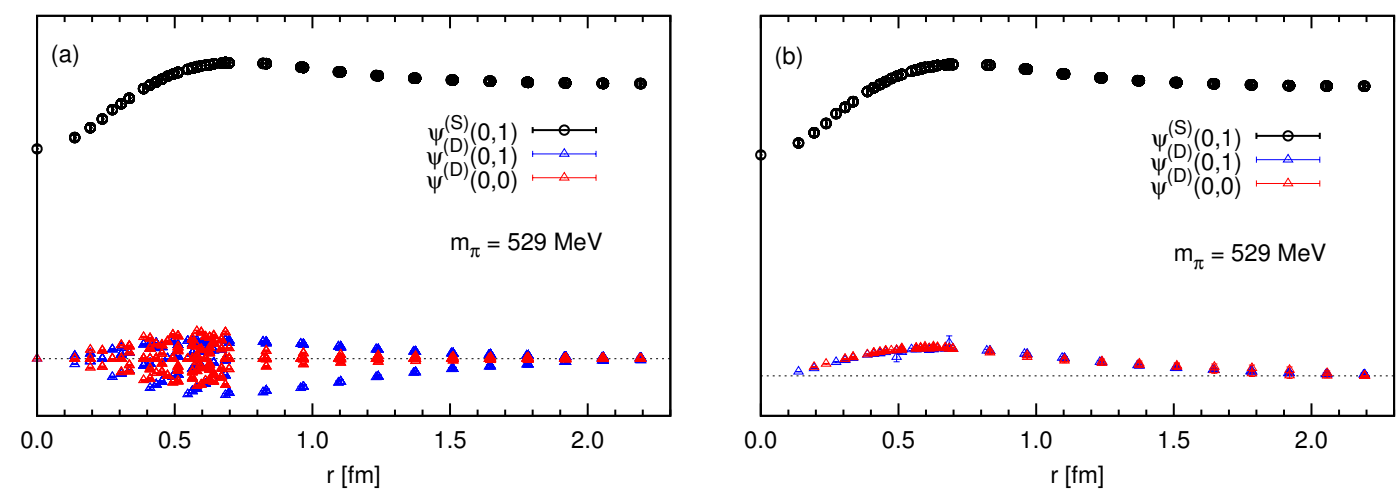

Figure 3: (a) BS wave functions on the lattice and (b) BS wave function after removing the spinor harmonics factors. 
component is proportional to the "spinor harmonics" in $\mathrm{SO}(3)$ symmetric limit as

$$
\left[\begin{array}{ll}
\psi_{00}(\vec{r}) & \psi_{01}(\vec{r}) \\
\psi_{10}(\vec{r}) & \psi_{11}(\vec{r})
\end{array}\right] \propto \mathbf{Y}_{J=1, M=0}^{(l=2)}(\hat{r}) \equiv \sqrt{\frac{3}{10}} \times\left[\begin{array}{cc}
Y_{2,-1}(\vec{r}) & -\frac{2}{\sqrt{6}} Y_{2,0}(\vec{r}) \\
-\frac{2}{\sqrt{6}} Y_{2,0}(\vec{r}) & Y_{2,+1}(\vec{r})
\end{array}\right]
$$

To examine whether $\psi^{(D)}$ is dominated by d-wave or not, we divide $\psi^{(D)}$ by these spinor harmonics factors. Fig. 3(b) shows the results. We see that $\psi^{(D)}$ becomes single valued, which indicates that $\psi^{(D)}$ is dominated by d-wave.

To separate the s-wave and the d-wave parts, we apply the projection operators $P$ and $Q$ to Eq. (3.4). Since $V_{C}(\vec{r})$ and $V_{T}(\vec{r})$ commute with $P$ and $Q$ due to the rotational invariance, we have

$$
\begin{aligned}
-\frac{\triangle}{m_{N}} P \psi_{\vec{k}}(\vec{r})+V_{C}(\vec{r}) P \psi_{\vec{k}}(\vec{r})+V_{T}(\vec{r}) P S_{12} \psi_{\vec{k}}(\vec{r}) & =\frac{k^{2}}{m_{N}} P \psi_{\vec{k}}(\vec{r}) \\
-\frac{\triangle}{m_{N}} Q \psi_{\vec{k}}(\vec{r})+V_{C}(\vec{r}) Q \psi_{\vec{k}}(\vec{r})+V_{T}(\vec{r}) Q S_{12} \psi_{\vec{k}}(\vec{r}) & =\frac{k^{2}}{m_{N}} Q \psi_{\vec{k}}(\vec{r}) .
\end{aligned}
$$

Note that each of these two equations has two spinor indices. For upper line, we have essentially a unique choice. $((0,1)$ and $(1,0)$ components agree to each other up to an overall sign.) In contrast, we can play with a particular choice of spin components for the lower line. Eq. (6.2) suggests that $(0,1)$ and $(1,0)$ component correspond to $E$-representation, whereas $(0,0)$ and $(1,1)$ component correspond to $T_{2}$-representation. Since $\mathrm{SO}(3)$ symmetry is not exact, the results depend on how we choose d-wave wave function. For simplicity in this section, we choose $(0,1)$ spin component from the lower line for d-wave. Eq. (6.3) is arranged as

$$
\left[\begin{array}{l}
P \psi_{\vec{k}}(\vec{r}), P S_{12} \psi_{\vec{k}}(\vec{r}) \\
Q \psi_{\vec{k}}(\vec{r}), Q S_{12} \psi_{\vec{k}}(\vec{r})
\end{array}\right] \cdot\left[\begin{array}{l}
V_{C}(\vec{r})-\frac{k^{2}}{m_{N}} \\
V_{T}(\vec{r})
\end{array}\right]=\left[\begin{array}{l}
\frac{\triangle}{m_{N}} P \psi_{\vec{k}}(\vec{r}) \\
\frac{\triangle}{m_{N}} Q \psi_{\vec{k}}(\vec{r})
\end{array}\right],
$$

which can be algebraically solved for $V_{C}(\vec{r})$ and $V_{T}(\vec{r})$ point by point. Unlike the central potential, the tensor potential does not involve an additional shift by $-\frac{k^{2}}{m_{N}}$, which adjusts zero at the spatial infinity. In Sect. 8, we employ another choice for the d-wave from the lower line by combining the four spinor components with the spinor harmonics $\mathbf{Y}(\hat{r}) \equiv \mathbf{Y}_{J=1, M=0}^{(l=2)}(\hat{r})$ as

$$
\mathbf{Y}_{\alpha \beta}^{*}(\hat{r})\left[Q \psi_{\vec{k}}\right]_{\alpha \beta}(\vec{r}) \cdot\left(V_{C}(\vec{r})-\frac{k^{2}}{m_{N}}\right)+\mathbf{Y}_{\alpha \beta}^{*}(\hat{r})\left[Q S_{12} \psi_{\vec{k}}\right]_{\alpha \beta}(\vec{r}) \cdot V_{T}(\vec{r})=\mathbf{Y}_{\alpha \beta}^{*}(\hat{r}) \frac{\triangle}{m_{N}}\left[Q \psi_{\vec{k}}\right]_{\alpha \beta}(\vec{r}) .
$$

Once $\mathrm{SO}(3)$ is realized as a good symmetry, this becomes the best choice.

Fig. 4(a) shows the results of the tensor potential together with the central and the effective central potentials. The shape of our tensor potential is similar to the one-boson exchange result, which is obtained by the cancellation between the pion exchange and the rho meson exchange [14]. The difference between the central and the effective central potentials is understood by treating the tensor potential in the second order perturbation theory. It seems to be smaller than phenomenologically expected. This is due to the heavy quark mass. Indeed, as is seen in Fig. 4(b), the tensor potential is enhanced in the light quark mass region.

A spike in the tensor potential at $r \simeq 0.5 \mathrm{fm}$ is due to the zero of $Y_{2,0}(\hat{r}) \propto 3 \cos ^{2} \theta-1$. Note that $Y_{2,0}(\hat{r})$ vanishes on the lines $\vec{r}=( \pm n, \pm n, \pm n)$. In the vicinity of these lines, it becomes difficult to solve the coupled equation numerically, which leads to the accumulated statistical error. 

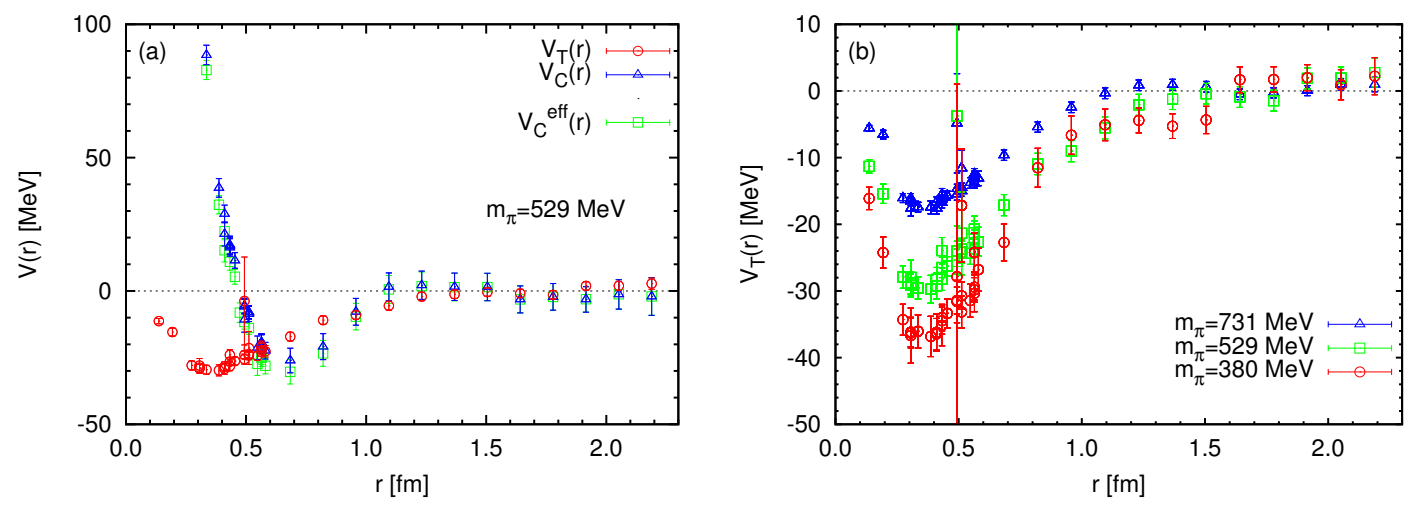

Figure 4: (a) The tensor, the central and the effective central potentials in ${ }^{3} S_{1}-{ }^{3} D_{1}$ coupled channel for $m_{p i}=529 \mathrm{MeV}$ and (b) the tensor potentials for three values of quark masses.

\section{Convergence of the derivative expansion}

We examine the convergence of derivative expansion by comparing two potentials generated at two energies $E \simeq 0 \mathrm{MeV}$ and $E \simeq 45 \mathrm{MeV}$ [15]. As soon as the derivative expansion is truncated, energy dependence may appear in the potential, because it is not in general possible to reproduce all the BS wave functions simultaneously only with truncated degrees of freedom. In this way, the energy dependence of the potential is related to the non-locality of the potential, which makes it possible to check the convergence of derivative expansion by examining the energy dependence.

We generate two potentials by imposing different spatial boundary conditions on quark fields. A potential at $E \simeq 0 \mathrm{MeV}$ is generated with the periodic boundary condition (PBC). A potential at $E \simeq 45 \mathrm{MeV}$ is generated with the anti-periodic boundary condition (APBC). With APBC, since each nucleon consists of odd number of quarks, a nucleon is also subject to APBC, so that its spatial momentum is discretized as $p_{i}=\left(2 n_{i}+1\right) \pi / L$ with $n_{i} \in \mathbb{Z}$. For a two nucleon system, the interaction (nuclear force) induces a modification from its free value, i.e., $p_{i}^{(r e l)} \simeq\left(2 n_{i}+1\right) \pi / L$. Note that the smallest spatial momentum for APBC is $\vec{p}^{(r e l)} \simeq( \pm \pi / L, \pm \pi / L, \pm \pi / L)$, which does not vanish. In the box with $L \simeq 4.4 \mathrm{fm},\left|\vec{p}^{(r e l)}\right| \simeq \sqrt{3} \pi / L=244 \mathrm{MeV}$. Comparison is made by using the setup with $m_{\pi}=529 \mathrm{MeV}, m_{N} \simeq 1333 \mathrm{MeV}$, which leads to the non-relativistic energy of the lowest-lying state $E \equiv k^{2} / m_{N} \simeq 45 \mathrm{MeV}$. The results are shown in Fig. 5. We see that the agreement is quite good except small deviations at short distance. The structures appearing in the region $r \gtrsim 1 \mathrm{fm}$ for APBC turn out to be caused by a small contamination of an excited state [15]. It follows that the derivative expansion works, and that our local potential constructed at the leading order can be reliably used in the energy region $E \simeq 0-45 \mathrm{MeV}$. (See Ref. [15] for detail.)

\section{2+1 flavor QCD result of nuclear force with PACS-CS gauge configuration}

In order to study the quantitative features of nuclear potentials, it is necessary to resort to dynamical QCD performed in the light quark mass region employing a large spatial volume. PACS-CS Collaboration is generating such gauge configurations, i.e., 2+1 flavor gauge configurations, which cover the physical quark mass employing large spatial volumes $L \sim 3-6 \mathrm{fm}$ 

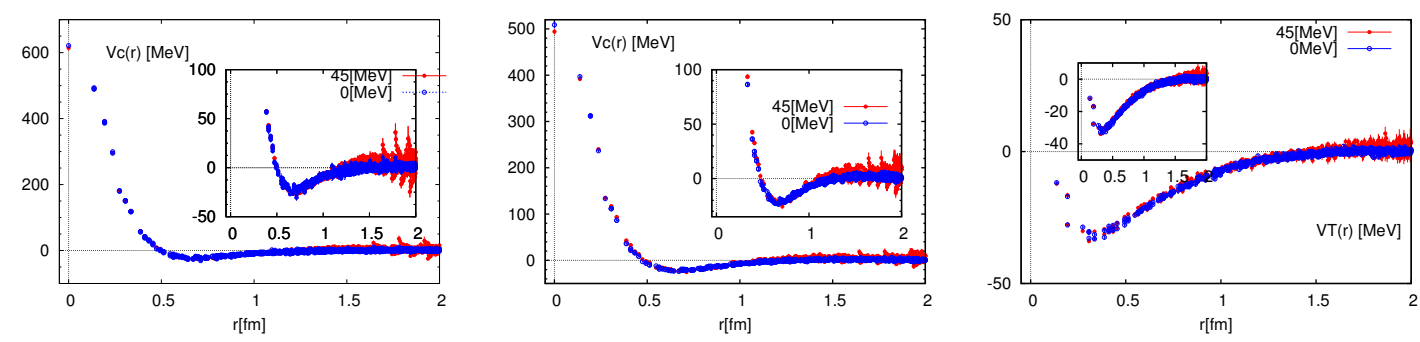

Figure 5: Comparisons of leading order potentials generated at two energies, i.e., $E \simeq 0 \mathrm{MeV}$ by $\mathrm{PBC}$ and at $E \simeq 45 \mathrm{MeV}$ by APBC. The left, middle, right figures show the central potential in ${ }^{1} S_{0}$ channel, the central and the tensor potentials in ${ }^{3} S_{1}-{ }^{3} D_{1}$ coupled channel, respectively.

[16]. We use PACS-CS gauge configurations to obtain 2+1 flavor QCD results of nuclear potentials. The gauge configurations are generated by employing Iwasaki gauge action at $\beta=1.90$ on $32^{3} \times 64$ lattice and $\mathrm{O}(a)$-improved Wilson quark (clover) action with a non-perturbatively improved coefficient $c_{\mathrm{SW}}=1.715 . m_{\pi}, m_{K}$ and $m_{\Omega}$ are used to determine the scale unit $a^{-1}=$ 2.176(31) $\mathrm{GeV}(a \simeq 0.091 \mathrm{fm}$ ) leading to the spatial extension $L=32 a \simeq 2.90 \mathrm{fm}$ [16]. To calculate nuclear potentials, we use three series of PACS-CS gauge configurations with $\left(\kappa_{u d}, \kappa_{s}\right)=$ $(0.16700,0.16400),(0.16727,0.16400)$ and $(0.16754,0.16400)$, which correspond to $m_{\pi} \simeq 701$, 570 and $411 \mathrm{MeV}$ and $m_{N} \simeq 1583,1412$ and $1215 \mathrm{MeV}$, respectively.
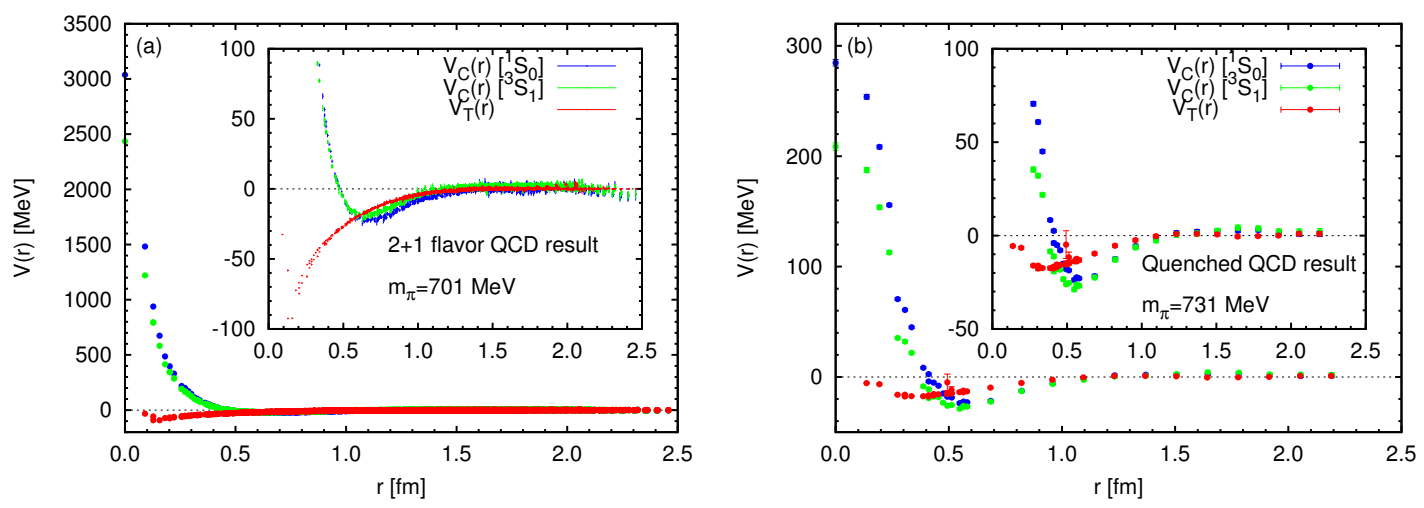

Figure 6: (a) 2+1 flavor QCD results of the central and tensor potentials for $m_{\pi}=701 \mathrm{MeV}$, and (b) quenched QCD results of the central and tensor potentials for $m_{\pi}=731 \mathrm{MeV}$.

Fig. 6(a) shows the $2+1$ flavor QCD results of the nuclear potentials for $m_{\pi} \simeq 701 \mathrm{MeV}$, which should be compared with the quenched results in Fig. 6(b) of comparable pion mass $m_{\pi} \simeq 731$ $\mathrm{MeV}$. We see that the repulsive cores at short distance and the tensor potential become significantly enhanced. The attraction at medium distance tends to be shifted to outer region, whereas it remains almost unchanged in magnitude. Although these changes may be caused by dynamical quarks, they may be due to a lattice discretization artifact. We need further information to conclude.

Fig. 7 shows the central potential $V_{C}(r)$ in ${ }^{1} S_{0}$ channel and the central potential $V_{C}(r)$ and the tensor potential $V_{T}(r)$ in ${ }^{3} S_{1}-{ }^{3} D_{1}$ coupled channel, together with those $r^{2}$ multiplied. Similar tendencies are observed as the quenched QCD, such as the enhancements of the repulsive cores at 

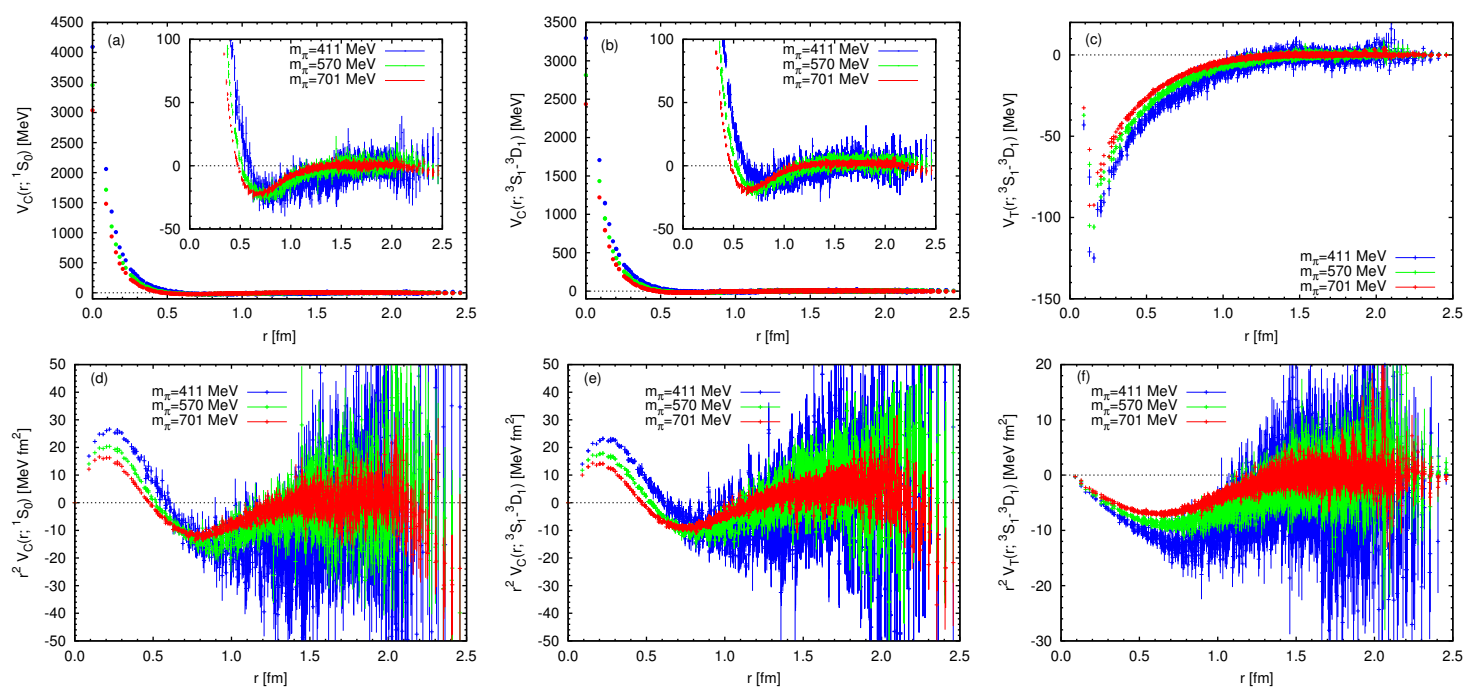

Figure 7: $2+1$ flavor QCD results of nuclear potentials for three values of quark mass. (a) $V_{\mathrm{C}}(r)$ in ${ }^{1} S_{0}$ channel, (b) $V_{\mathrm{C}}(r)$ in ${ }^{3} S_{1}-{ }^{3} D_{1}$ coupled channel, (c) $V_{\mathrm{T}}(r)$ for ${ }^{3} S_{1}-{ }^{3} D_{1}$ coupled channel, (d) $r^{2} V_{\mathrm{C}}(r)$ in ${ }^{1} S_{0}$ channel, (e) $r^{2} V_{\mathrm{C}}(r)$ in ${ }^{3} S_{1}-{ }^{3} D_{1}$ coupled channel, (f) $r^{2} V_{\mathrm{T}}(r)$ for ${ }^{3} S_{1}-{ }^{3} D_{1}$ coupled channel.

short distance, the attractive pockets at medium distance, and the strength of the tensor potential.


Figure 8: (a) Scattering phase shifts in ${ }^{1} S_{0}$ channel from the lattice nuclear potentials and (b) those with the empirical one.

Fig. 8 shows the phase shifts obtained from the nuclear potentials (not by Lüscher's method). At low energy, the phase shift grows up, which is caused by the attraction at medium distance. At high energy, the phase shift decrease, which is a consequence of the repulsive core at short distance. One may wonder why the order of the phase shifts of $m_{\pi}=570$ and $701 \mathrm{MeV}$ is inverted. The reason seems to be that the repulsive core grows more rapid than the attraction grows. Qualitative shape of the phase shift is seen to be reasonable, which is due to the fact that the qualitative features of the nuclear potential are already reproduced. However, comparing with the empirical one, the strength is not satisfactory at all, which suggests the importance of the light quark mass effect.

A technical comment is in order. To obtain the scattering length, the ground state saturation 
has to be achieved to an accuracy of, at most, around $1 \mathrm{MeV}$, which is about $0.05 \%$ of the total mass of the two nucleon system. For the moment, such a high precision is not yet attained in our calculation. Significantly large $t$ and, accordingly, the large statitics are required. To calculate only the scattering length, the smeared source is better than the wall source for such high precision calculations. The smeared source, with the help of the average over the relative coordinate $\vec{x}$ in the sink side, projects out the excited state contamination from the temporal correlation. However, to calculate the BS wave function, which measures the spatial correlation, the sink has to be unaveraged, and the ground state saturation has to be achieved point by point uniformly in the spatial directions. Note that the smeared source creates a spatially squeezed BS wave function in the small $t$ region, which gradually broadens during the temporal evolution, until the ground state shape is achieved. Here, the convergence in the region $|\vec{x}| \gtrsim 1 \mathrm{fm}$ is quite slow, and unreasonably large $t$ is required for the uniform saturation by the ground state. For future applications to the nuclear physics, it is necessary to seek for a better source, which makes it possible to achieve the uniform saturation of the ground state BS wave function more efficiently.

\section{Hyperon potentials}

Hyperon potentials (hyperon-nucleon and hyperon-hyperon) serve as the starting point in studying the hyper-nuclei structure. They have large influence on the hyperon matter generation in neutron star core. In spite of their importance, we have only a limited knowledge of the hyperon potentials, because of the lack of experimental information. Since we do not need any information from the scattering experiment, we apply our method to construct the hyperon potentials. The first attempts have been made to construct $N \Xi(I=0)$ potentials and $N \Lambda$. The results are shown in Fig. 9 (See Refs. [17, 18] for detail.) These calculations are being extended to $N \Sigma$ and $\Lambda \Lambda$ potentials. It
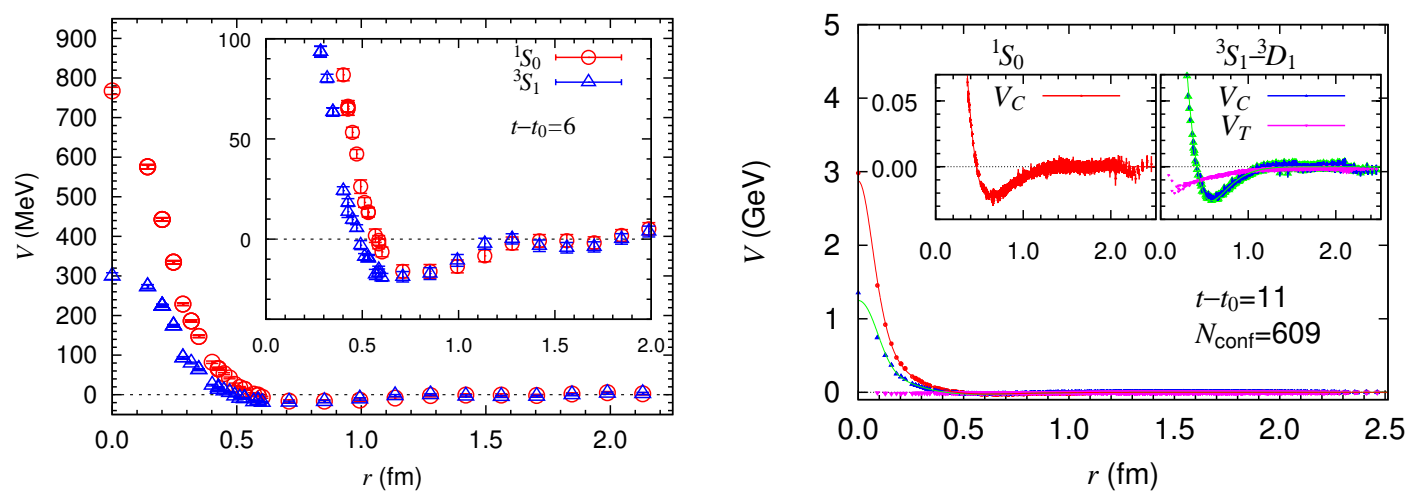

Figure 9: $N \Xi$ potentials $(I=1)$ from quenched QCD (left) and $N \Lambda$ potentials from $2+1$ flavor QCD (right).

is important to examine the convergence of the derivative expansion. After this process, the reliability of the potential is guaranteed in the well-defined applicability region. Such potentials may be used as alternatives to experimentally constructed potentials, before experimental information on various hyperon scatterings becomes fully available.

Hyperon potentials give us another benefit. Their flavor structure is expected to provide us with an important key to unveil the physical origin of the repulsive core. To obtain a simplified 
picture, investigations in the flavor SU(3) idealized limit would be useful and interesting. Works are in progress along this line [19]. It would be also interesting to compare these numerical results with analytical ones obtained by the operator product expansion [20].

\section{Summary}

We have reported lattice study of nuclear potentials based on the equal-time Bethe-Salpeter (BS) wave function for two nucleon system. We have defined the nuclear potential by the effective Schrödinger equation demanding that it should generate the BS wave functions in wide range of energy region simultaneously. The method generates realistic nuclear potentials, because of the remarkable similarity in the asymptotic behaviors between the BS wave function of QCD and the non-relativistic wave function of scattering state in the quantum mechanics. The central and the tensor potentials have been obtained at the leading order of the derivative expansion, which show the qualitative features of the phenomenological nuclear potentials. The convergence of the derivative expansion has been examined by comparing two potentials generated at different energies. We have found that the discrepancy is small in the low energy region $0 \lesssim E_{\mathrm{CM}} \lesssim 45$ $\mathrm{MeV}$, which indicates that the derivative expansion works. For quantitative applications to nuclear physics, 2+1 flavor QCD should be used to generate the nuclear potentials in the light quark mass region. By using PACS-CS gauge configurations, we have attempted to obtain $2+1$ flavor QCD results of the nuclear potentials. Qualitative features remains the same except for the enhancements of the repulsive core, the range of the attraction of the central potentials, and the strength of the tensor potential. These nuclear potentials have been used to calculate the phase shifts, which behave reasonably. Although they are reasonable in a qualitative sense, their strength is not satisfactory at all, which suggests the importance of the lattice QCD calculation in the light quark mass region. Finally, we have applied our method to the hyperon potentials such as $N \Xi$ and $N \Lambda$, for which only a limited number of experimental information is available for the moment.

It is interesting to use our nuclear potentials to study the nuclear many body problems, which provides a way to access nuclei based on QCD. Needless to say, there is another direct way to access nuclei by lattice QCD, i.e., direct calculations of nuclear spectrum, matrix elements, etc [21]. These two approaches are considered to be complementary. The former keeps a connection to the conventional nuclear theory, while a number of formalisms and techniques have to be established. The latter loses a connection to the conventional nuclear theory, while many of the existing techniques in lattice QCD can be used. It is desirable to use both of these two approaches as the situation demands. All that is certain is that the lattice QCD will provide a unique tool to study realistic nuclei in the quite near future.

\section{Acknowledgments}

Quenched QCD Monte Carlo calculations have been performed with IBM Blue Gene/L at KEK under the "Large scale simulation program" at KEK (No. 09-23). 2+1 flavor lattice QCD Monte Carlo calculations have been done with the super computer PACS-CS and T2K under the "Interdisciplinary Computational Science Program" of Center for Computational Science at University of Tsukuba (No 09a-11). We are grateful for the authors and maintainers of $C P S++[22]$, 
of which a modified version is used for measurement done in this work. This work was partly supported by Grant-in-Aid of the Japanese Ministry of Education, Science and Technology, Sports and Culture (Nos. 19540261, 20340047 ), Scientific Research on Priority Areas (No. 20028013), Scientific Research on Innovative Areas (Nos. 20105001, 20105002, 20105003, 21105515), and Specially Promoted Research (No. 13002001).

\section{References}

[1] V.G.J. Stokes, R.A.M. Klomp, C.P.F. Terheggen and J.J. de Swart, Phys. Rev. C 49, 2950 (1994). R.B. Wiringa, V.G.J. Stokes and R. Schiavilla, Phys. Rev. C 51, 38 (1995).

R. Machleidt, Phys. Rev. C 63, 024001 (2001).

[2] S. Weinberg, Phys. Lett. B 251, 228 (1990); Nucl. Phys. B 363, 3 (1991). P.F. Bedaque and U. van Kolck, Ann. Rev. Nucl Part. Sci. 52, 339 (2002).

R. Machleidt, arXiv:0704.0807[nucl-th].

E. Epelbaum, H.W. Hammer and U.G. Meissner, Rev. Mod. Phys. 81, 1773 (2009).

[3] M. Lüscher, Nucl. Phys. B 354, 531 (1991).

[4] M. Fukugita, Y. Kuramashi, M. Okawa, H. Mino and A. Ukawa, Phys. Rev. D 52, 3003 (1995).

[5] S.R. Beane, P.F. Bedaque, K. Orginos and M.J. Savage, Phys. Rev. Lett. 97, 012001 (2006).

[6] S. Muroya, A. Nakamura, J. Nagata, Nucl. Phys. B(Proc. Suppl.) 129, 239 (2004).

S.R. Beane et al., NPLQCD Coll., Nucl. Phys. A 794, 62 (2007).

S.R. Beane et al., NPLQCD Coll., arXiv:0912.4243[hep-lat].

[7] N. Ishii, S. Aoki and T. Hatsuda, Phys. Rev. Lett. 99, 022001 (2007).

[8] C.-J.D. Lin, G. Martinelli, C.T. Sachrajda and M. Testa, Nucl. Phys. B619, 467 (2001).

[9] S. Aoki et al., CP-PACS Coll., Phys. Rev. D 71, 094504 (2005).

[10] S. Aoki, T. Hatsuda and N. Ishii, Comput. Sci. Dis. 1, 015009 (2008).

S. Aoki, T. Hatsuda and N. Ishii, arXiv:0909.5585[hep-lat].

[11] K. Nishijima, Phys. Rev. 111, 995 (1958).

W. Zimmermann, Nuovo Cim. 10, 597 (1958).

R. Haag, Phys. Rev. 112, 669 (1958).

[12] S. Okubo and R.E. Marshak, Ann. Phys. 4, 166 (1958).

[13] R. Subedi et al., Science 320, 1476 (2008).

[14] R. Machleidt, Adv. Nucl. Phys. 19, 189 (1989).

[15] K. Murano, N. Ishii, S. Aoki and T. Hatsuda for HALQCD Coll., in these proceedings. K. Murano et al., HALQCD Coll., in preparation.

[16] S. Aoki et al., PACS-CS Coll., Phys. Rev. D 79, 034503 (2009).

[17] H. Nemura, N. Ishii, S. Aoki and T. Hatsuda, Phys. Lett. B673, 136 (2009).

[18] H. Nemura for HALQCD Coll., in these proceedings.

[19] T. Inoue for HALQCD Coll., in these proceedings.

[20] S. Aoki, J. Balog and P. Weisz., in these proceedings.

[21] T. Yamazaki, Y. Kuramashi and A. Ukawa, PACS-CS Coll., arXiv:0912.1383[hep-lat].

[22] CPS++ http://qcdoc.phys.columbia.edu/chuiwoo_index.html (maintainer: Chulwoo Jung). 